\title{
Exploring Usability of School Closure Data for Influenza-Like IIIness Surveillance
}

\author{
Yenlik Zheteyeva*, Hongjiang Gao, Jianrong Shi and Amra Uzicanin \\ Centers for Disease Control and Prevention, Atlanta, GA, USA
}

\section{Objective}

Evaluate usability of alternative data sources, such as public announcements of unplanned school closures, for additional insight regarding influenza-like illness (ILI) activity.

\section{Introduction}

School children are the primary introducers and significant transmission sources of influenza virus among their families and surrounding communities [1,2]. Therefore, schools play an important role in amplifying influenza transmission in communities. Using school-related data sources may be an informative addition to existing influenza surveillance. Unplanned school closures (USCs) are common, occur frequently for various reasons, and affect millions of students across the country [3]. Information about USCs is publicly available in real-time. For this study, we evaluated usability of applying USC data for ILI surveillance.

\section{Methods}

From August 1, 2011 through June 30, 2015, we conducted systematic daily searches of publicly available online data (via Google, Google News, and Lexis-Nexis) to identify USCs lasting $\geq 1$ school day in the United States. We selected USCs for which infectious diseases, particularly respiratory illnesses, were indicated as the main reason for the school closures. We described these USCs and compared their temporal patterns with ILI data from outpatient provider visits (available via ILINet at http://gis.cdc.gov/grasp/ fluview/fluportaldashboard.html). We also evaluated the correlation (at alpha $=0.05$ ) between weekly occurrence of ILI, reported by ILINet, and respiratory illness-related USCs over the 4 school years, excluding summer breaks (weeks 26-31).

\section{Results}

Of the 396 USCs related to infectious diseases, 232 (59\%) were due to respiratory illnesses; the duration of these closures ranged from 1-4 days (based on 135 USC for which these data were available). The patterns of respiratory illness-related USCs corresponded similarly with those of ILI activity observed via ILINet data regardless of the severity of influenza season (Figure 1). During the 2012-13 and 2014-15 influenza seasons, when ILI activity was high and peaked at around $6 \%$, the number of USCs nationwide peaked as well; combined, these 2 school years accounted for $191(82.3 \%)$ of all respiratory illness-related USCs over the 4-year study period. In 2012-13, ILI activity was highest around the winter holiday break (at weeks 51-52). Following this winter break, a small increase in ILI activity at week 4 corresponded with a peak in USCs: more than 30 USCs were announced. Similar patterns characterized the 2014-15 influenza season. In 2011-12 and 2013-14 when the influenza seasons were milder and ILI activity lesser, USC patterns still corresponded to those of ILI activity with highest increase in USCs on week 6 in 2012 and on week 2 in 2014. Overall correlation between USC and ILINet data was significant: $\mathrm{R}=0.54$ with $\mathrm{p}$-value $<0.0001$.

\section{Conclusions}

Our data demonstrated that the occurrence pattern of respiratory illness-related USCs is reflective of the national pattern of ILI activity. This suggests that monitoring USCs can be a useful addition to existing influenza surveillance systems, particularly useful during severe influenza seasons when respiratory illness-related USCs may occur more frequently. Also, other studies of USCs [4] indicated that the main reason for respiratory illness-related closures was increased student absenteeism; thus, usability of cause-specific student absenteeism data for monitoring local and state influenza activity should be evaluated via prospective studies.

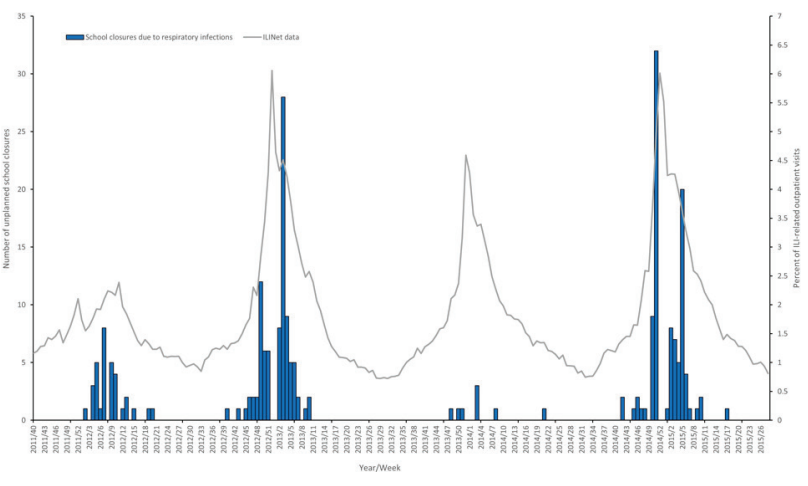

Figure 1 Respiratory illness-related USCs and percentage of outpatient provider visits for ILI in the United States by week, August 2011 - June 2015

Note: Weeks 26-31 correspond with school summer breaks

\section{Keywords}

School closures; influenza; surveillance

\section{References}

Longini IM, Jr., Koopman JS, Monto AS, Fox JP. Estimating household and community transmission parameters for influenza. $\mathrm{Am} J$ Epidemiol. 1982 (115).

Viboud C, Boelle PY, Cauchemez S, et al. Risk factors of influenza transmission in households. Br J Gen Practice. 2004 (54).

Wong K, Shi J, Gao H et al. Why is the school closed today? Unplanned K-12 school closures in the United States, 2011-2013. PLoS One December 2, 2014.

Johnson AJ, Moore ZS, Edelson PJ, et al. Household responses to school closure resulting from outbreak of influenza B, North Carolina. Emerg Infect Dis. 2008;14(7).

\section{*Yenlik Zheteyeva}

E-mail: yzheteyeva@cdc.gov 